\title{
Indicadores da escolha de Pediatria por graduandos da Universidade de Brasília: estudo longitudinal em série histórica
}

\author{
Factors associated with Pediatrics as a career \\ choice by undergraduate medical students: a \\ longitudinal study of a historical series
}

Dejano Tavares Sobral ${ }^{\mathrm{I}}$

Dioclécio Campos Júnior ${ }^{\mathrm{I}}$

\author{
PALAVRAS-CHAVE \\ - Educação de Graduação em \\ Medicina \\ - Internato e Residência \\ - Escolha de profissão \\ - Pediatria
}

\begin{abstract}
R E S U M O
Os autores analisaram a escolha de residência médica em Pediatria dentre 877 egressos do curso de Medicina da Universidade de Brasília, formados no período 1994-2007. Os dados abrangeram descritores demográficos e de aprendizagem, preferência inicial por carreira, monitoria, rendimento acadêmico, estágio seletivo no internato e inscrição para residência médica. Efetuaram-se análises de contingência e de regressão logística agrupando-se os egressos por escolha de Pediatria ou não. Do total, 9,7\% escolheram Pediatria, dentre os quais 44,7\% mostraram atração inicial e 45,9\% expressaram interesse pela área já na fase clínica. No período 2002-2007, houve declínio significante da escolha, em conexão com menor procura por monitoria e estágio seletivo na área, mas sem tendência temporal na atração inicial. A regressão logística identificou cinco fatores preditivos, nesta ordem decrescente de magnitude de efeito na escolha: estágio seletivo, rendimento global, estilo de aprendizagem reflexivo, ordem de preferência inicial e época da graduação. Os efeitos independentes de sexo e monitoria não foram significantes. Concluindo, os fatores preditivos da escolha de Pediatria incluíram indicadores pessoais e curriculares em vertentes distintas de interesse e influência, num quadro de queda recente da opção pela área.
\end{abstract}

\section{A B S T R A C T}

The authors analyzed Pediatrics as career choice in a study involving 877 graduates from the University of Brasilia from 1994 to 2007. Data included demographics, learning attributes, early career preference, peer-tutoring experience, academic achievement, selective training in the last semester, and choice of residency training. Contingency and logistic regression analyses were performed with the graduates grouped according to choice of Pediatrics versus all other options. Overall, $9.7 \%$ of graduates had chosen Pediatrics, of whom $44.7 \%$ had indicated early attraction to the specialty, while another $45.9 \%$ did not express interest until their clinical training. No significant trend was shown for early attraction, but a lower proportion of graduates chose Pediatrics in the last 6 years, in parallel with fewer applications for tutoring experience and selective training. Logistic regression identified five predictors of choice in decreasing order of effect size: selective training, overall achievement, reflective learning style, ranking of early preference, and time of graduation. Independent effects of gender and peer tutoring were not statistically significant. In conclusion, the predictors of choice of Pediatrics comprised personal and curricular features in distinct patterns of interest and influence, within an overall context of decreasing choice of the specialty in recent years. 


\section{INTRODUÇÃO}

Quais são as características pessoais e de vivência no curso associadas à escolha de carreira em Pediatria e sua consistência ao longo dos anos? O caminho educacional dos estudantes no processo de escolha de carreira durante o curso de Medicina tem significância educacional e impacto social, uma vez que a opção profissional dos recém-graduados afeta a composição, distribuição geográfica e estratificação nos níveis de atenção à saúde provida pela força de trabalho médico. No caso da Pediatria, distorções nas expectativas iniciais e desencontros no caminho educacional e na escolha do treinamento especializado têm consequências potenciais para o acesso de uma camada singular da população a profissionais qualificados, bem como para a cobertura da assistência médica nos distintos níveis de sua organização.

Estudos significativos nos últimos 20 anos têm delineado a natureza multidimensional das influências sobre a escolha da área de especialização, em residência médica, por egressos do curso de Medicina ${ }^{1-3}$. Essas influências comportam os fatores que antecedem o treinamento clínico, os decorrentes do treinamento clínico e aqueles prospectivos, oriundos da antevisão das perspectivas da inserção profissional no campo de trabalho. Os fatores são parcialmente condicionados pelas injunções do campo de trabalho, incluindo as demandas específicas de grupos da população, o filtro de acesso determinado pela oferta de vagas para ingresso na residência médica em diferentes especialidades, assim como os desafios, as oportunidades e recompensas da ocupação funcional ${ }^{4}$. Há, contudo, evidências, tais como o perfil pessoal variável e a intensidade das preferências precoces, que indicam um substrato consistente de diferenças individuais em aptidões e personalidade influenciando a escolha da área profissional ${ }^{5}$.

Assinalem-se, também, as influências potenciais da escola médica - na confirmação, ou eliminação da preferência inicial e inclusão de preferência nova -, conforme realçado noutros estudos $^{6-9}$. As influências abrangem múltiplos aspectos da ambiência institucional, incluindo cenários de formação, currículo e recursos humanos e talvez afetem a estabilidade relativa das preferências iniciais entre as várias especialidades ${ }^{10}$. Elas incidem principalmente durante a vivência clínica, na forma de disciplina prática ou estágio de treinamento, de caráter obrigatório ou optativo, em diferentes cenários de atendimento. Envolvem tanto a adequação da supervisão e imagem profissional dos preceptores dos aprendizes, quanto a reatividade destes últimos no que concerne à compatibilidade pessoal com atributos e habilidades requeridos no trato de pacientes da área de treinamento ${ }^{10-12}$.
Vários estudos examinaram diretamente fatores de influência na escolha de Pediatria por estudantes de Medicina. $O$ fator sexo foi realçado em estudos no Brasil e no exterior, os quais apontam a predominância de mulheres na preferência por Pediatria do início à conclusão do curso ${ }^{4,13-14}$. Claramente, a vinculação com a faixa etária (lidar com crianças e adolescentes) é um determinante crítico da preferência ${ }^{15-18}$. Esse vínculo, aliás, é um de dois ou três aspectos distintivos realçados em textos de orientação para escolha da especialidade, dirigido aos estudantes de Medicina ${ }^{19,20}$. Características psíquicas, relacionadas às dimensões da personalidade, parecem influenciar a escolha, na medida em que sugerem um perfil distintivo do pediatra, ainda que os achados de diferentes estudos não sejam conclusivos ${ }^{5,21-24}$. Há indícios, também, de que o estilo individual de aprendizagem influencia a preferência por Pediatria ou outra área durante o curso ${ }^{24,25}$. Acrescentem-se, na perspectiva da influência educacional, as indicações de que uma experiência estimulante e encorajadora no treinamento clínico aumenta a adesão à especialidade. A exposição a profissionais exemplares pode contribuir para essa adesão, especialmente quando o ambiente de treinamento é favoráve ${ }^{26-29}$.

Em suma, as indicações referidas sugerem uma combinação de fatores pessoais e vivenciais influenciando a escolha de Pediatria. Entretanto, a maioria dos estudos publicados tinha foco transversal ou exclusivamente retrospectivo, e poucos examinaram a interação entre os fatores identificados.

O presente trabalho faz parte de um programa de estudo sobre fatores de influência na manutenção ou alteração das preferências dos estudantes na escolha da carreira, segundo um acompanhamento longitudinal de coortes consecutivas do curso de Medicina da Universidade de Brasília (UnB). Um trabalho inicial revelou efeitos preditivos diferenciais de sexo, preferência inicial e área de estágio seletivo na escolha da residência médica por graduandos do primeiro quadriênio após a renovação curricular, há 20 anos $^{30}$. O incentivo para a divulgação da análise atual veio da antevisão de desequilíbrio potencial na situação de demanda e oferta de profissionais na área de Pediatria. Consideram-se as alterações nos perfis demográfico, cultural e nosológico da população, as crescentes exigências na formação profissional e nas condições de trabalho, bem como a ampliação de opções de atuação profissional. Em tese, estas mudanças poderiam afetar as expectativas do próprio alunado em relação aos desafios e recompensas de uma carreira na especialidade. Um levantamento realizado na UnB, aliás, revelou declínio significante na atração inicial por Pediatria no quinquênio 2004-2008.

O propósito deste artigo é analisar a escolha de residência médica em Pediatria em função de atributos pessoais e indicado- 
res curriculares e na perspectiva de uma série histórica, após a mudança curricular naquele curso. Três objetivos guiam a apresentação:

- identificar características de entrada e de vivência no curso que relacionam interesse e/ou preferência por Pediatria com a escolha da especialidade, entre os egressos;

- verificar os fatores associados à confirmação ou mudança de preferência: a eliminação ou a inclusão de Pediatria como opção, entre o início e o término do curso;

- estimar, mediante análises de regressão logística, a contribuição relativa das diversas características e da época de graduação como fatores preditivos da escolha dessa área profissional no período de 1994 a 2007.

\section{CASUÍSTICA E MÉTODOS}

\section{Contexto}

No período do estudo, a graduação médica na Universidade de Brasília foi conformada pela renovação curricular implantada a partir do segundo semestre de 1988. Os quatro seguintes elementos do novo acompanhamento curricular são realçados: (a) formação clínica no Hospital Universitário de Brasília (HUB) a partir do quinto semestre do curso; (b) realização do internato em três semestres, sendo os primeiros em rodízio nas quatro clínicas básicas e o terceiro semestre em caráter seletivo, dentre as áreas de Clínica Médica, Clínica Cirúrgica, Pediatria, Ginecologia e Obstetrícia, ou Medicina Geral e Comunitária; (c) amplo acesso a disciplinas optativas em áreas pré-clínicas e clínicas; (d) múltiplas oportunidades de realização de monitorias em disciplinas obrigatórias ou optativas com obtenção de créditos para integração curricular. As atividades obrigatórias diretamente relacionadas à Pediatria incluem, além do internato, três disciplinas oferecidas no oitavo e nono períodos do currículo.

O curso de Medicina, até 1999, fazia parte da Faculdade de Ciências da Saúde, da qual se separou com a criação da Faculdade de Medicina na Universidade de Brasília.

\section{População-alvo}

O estudo incluiu todos os egressos das 28 primeiras turmas semestrais do novo acompanhamento curricular no período 1994-2007. O acesso ao curso deu-se por processo interno na UnB (exame vestibular e, mais recentemente, avaliação seriada) para $86,1 \%$ dos egressos ou mediante processo externo (transferência de outras escolas e matrícula cortesia de estrangeiro) para os demais $13,9 \%$. O número anual de egressos variou de 40 a 85 no período de 14 anos, passando da mediana de 59 na primeira meta- de para 70 na segunda metade do período. Do total de 877 graduandos, $45,6 \%$ eram mulheres e $24,4 \%$ concluíram o curso com 23 anos ou menos de idade.

\section{MÉTODOS}

Os dados do estudo derivam de três fontes principais: (1) questionário de expectativas, preenchido pelos estudantes no início do segundo ano do curso e que apurava a preferência por carreira e os descritores de aprendizagem; (2) registro no curso, incluindo forma de admissão, dados demográficos e de histórico escolar; (3) registro de inscrição e seleção em programas de residência médica em serviços públicos de saúde no País no período 1994-2007. Os dados do questionário ficaram incompletos no caso de $12(1,37 \%)$ egressos.

Três grupos de indicadores foram identificados, como descrito a seguir.

- Características de entrada. As variáveis selecionadas foram sexo, idade, naturalidade, estilo de aprendizagem, autoconfiança como aprendiz, preferência por carreira ao ingressar na UnB e forma de acesso ao curso. As respostas dos participantes ao inventário de estilo de aprendizagem de Kolb, primeira versão, definiram dois enfoques de aprendizagem: ativo (estilos convergente e adaptativo) e reflexivo (estilos divergente e assimilador $)^{31}$.

- Características relacionadas ao curso. As variáveis selecionadas foram preferência por carreira após o primeiro ano de curso, índices de rendimento da aprendizagem, monitorias em Pediatria ou noutras áreas e opção de estágio seletivo no terceiro semestre do internato.

O questionário de expectativas solicitava aos participantes uma ordenação das opções de carreira na escala ordinal de quatro postos de preferência: primeira, segunda, terceira ou preferência indeterminada, bem como o esclarecimento dos motivos para a primeira preferência. A expressão de primeira preferência ao ingressar no curso e/ou após o primeiro ano médico caracterizou o indicador de atração inicial por Pediatria.

Quanto aos produtos da aprendizagem, foram apurados os índices de rendimento acadêmico no curso (rendimento global) e nas disciplinas de Pediatria. Calculou-se também o quociente do índice médio nas disciplinas de Pediatria dividido pelo índice médio nas disciplinas de Cirurgia. Este rendimento relativo foi codificado como variável contínua (razão de rendimento) e como variável ordinal binária (nível de rendimento), usando a mediana do escore. 
- Desfecho. A primeira inscrição em programa de residência médica de acesso direto definiu a variável binária de desfecho, codificada como escolha de Pediatria, ou escolha de qualquer outra opção. No caso de inscrição para mais de uma especialidade, valeu aquela que resultou na seleção ou superior classificação do egresso.

\section{Análise estatística}

Duas referências orientaram a análise estatística ${ }^{32,33}$. Os principais procedimentos utilizados foram: (a) testes para aferir associação ou correlação entre indicadores potenciais e a variável de desfecho; (b) testes t, ou análises de variância, para aferir diferenças entre médias de grupos; (c) análises de regressão logística (LR, stepwise) para examinar a associação entre fatores selecionados e a variável de desfecho. O nível de significância foi alfa $=0,05$ (two-sided). Utilizou-se o programa SPSS versão 9.0 na análise.

\section{RESULTADOS}

Dentre os 877 egressos, 9,7\% (85) escolheram Pediatria na inscrição para primeira residência médica, uma proporção significantemente menor (teste de McNemar $\mathrm{p}=0,002$ ) do que os $14,0 \%$ que indicaram preferência principal pela área ao ingressarem no curso. A diferença entre as proporções de homens (6,1\%) e mulheres $(14,3 \%)$ na escolha foi muito significante $(p<0,001)$. A diferença na distribuição sexual era ainda mais expressiva na preferência pela especialidade por ocasião do ingresso no curso (7,4\% de homens v. $21,8 \%$ de mulheres; $\mathrm{p}<0,001)$. Não houve tendência significante de alteração na preferência inicial por Pediatria dentre os participantes das coortes consecutivas (qui ao quadrado para tendência $=2,70 \mathrm{df}=1 \mathrm{p}=0,10$ ). Entretanto, observou-se uma tendência significante de declínio na proporção dos egressos que escolheram a especialidade na residência (qui ao quadrado para tendência $=10,3 \mathrm{df}=1 \mathrm{p}<0,001$ ), definida a partir do quinto biênio no período.

Os participantes, ao término do primeiro ano do curso, justificaram a preferência principal por Pediatria com razões variadas. Em suma, os motivos apontados para a preferência pela carreira podem ser agrupados em quatro maiores categorias: [a] gostar de crianças e de lidar com crianças; [b] (a carreira) ensejar disposição e oportunidades de ajudar; [c] receptividade a influência externa significante; e [d] ter expectativas de realização ou identificação pessoal na especialidade. As duas primeiras indicações foram mais frequentes entre aqueles que confirmaram a preferência por Pediatria na escolha da residência, enquanto as duas últimas predominaram entre os que eliminaram essa opção inicial quando da escolha da residência.
A Tabela 1 mostra os dados para as medidas de associação (coeficiente Phi e razão de chance ou odds ratio) entre características de entrada ou de vivência no curso e a escolha de Pediatria para a totalidade dos egressos. Foram observadas associações significantes entre a opção por Pediatria e as nove seguintes características, em ordem crescente de magnitude: rendimento relativo maior, rendimento global superior, estilo de aprendizagem reflexivo, época da graduação (1994-2001), sexo feminino, preferência por Pediatria ao ingressar no curso, preferência por Pediatria após o primeiro ano do curso, monitoria e estágio seletivo nessa área. Não houve associação significante entre a escolha de Pediatria e a autoconfiança como aprendiz, a naturalidade ou a faixa etária do graduando. Note-se o expressivo valor preditivo positivo $(0,63)$ da opção de estágio seletivo para a escolha de Pediatria.

Quatro características (preferência por ocasião do ingresso, preferência após o primeiro ano, realização de monitoria e estágio seletivo na área) definem uma sequência de indicadores de interesse por Pediatria na trajetória da graduação. A ordem temporal ajudou a identificar o evento da primeira concordância entre a escolha de residência em Pediatria e a instância prévia de interesse ou preferência. Tal critério permitiu classificar os 85 egressos que escolheram a área em cinco grupos de concordância. Para quase a metade, a primeira concordância surgiu antes da fase clínica; assim, 32 deles (37,6\%) expressaram a primeira concordância na indicação de preferência ao ingressarem na universidade e $6(7,1 \%)$ após o primeiro ano do curso. Outros 12 egressos $(14,1 \%)$ a expressaram após a iniciação clínica, mediante realização de monitoria na área. Já para $27(31,8 \%)$, a primeira concordância ocorreu no internato, na opção por estágio seletivo em Pediatria. Finalmente, para 8 (9,4\%) não ocorreu evento de concordância anterior à escolha de residência. Observou-se tendência de predominância de sexo feminino na concordância precoce de indicação de interesse com escolha da especialidade $(P h i=0,319 \mathrm{p}=0,70)$.

Apurou-se uma medida de interesse iterativo em Pediatria somando-se a pontuação de ocorrência ( 1 = sim, 0 = não) dos quatro indicadores: preferência por ocasião do ingresso, preferência após o primeiro ano, monitoria em Pediatria e estágio seletivo na área. Essa medida de interesse mostrou coeficiente de correlação (tau-b) significante com sexo feminino (tau- $b=0,212 p<0,001$ ), estilo de aprendizagem reflexivo (tau- $b=0,073 p=0,024$ ) e escolha de residência em Pediatria (tau- $b=0,469 p<0,001$ ). Os dados constam da Tabela 2. Consistentemente, o coeficiente de correlação entre medida de interesse e índice de rendimento em Pediatria foi positivo e significante (rho $=0,140 \mathrm{p}<0,001$ ). 


\section{Tabela 1}

Medidas de associação entre as características de ingresso e de seguimento no curso e a escolha de residência médica em Pediatria (Ped.) por egressos de Medicina da Universidade de Brasília ( $\mathrm{N}=877$ )

\begin{tabular}{|c|c|c|c|c|c|}
\hline \multirow{2}{*}{$\begin{array}{l}\text { Indicadores } \\
\text { Sequência temporal }\end{array}$} & \multirow{2}{*}{$\begin{array}{l}\text { Escolha } \\
\text { Pediatria }\end{array}$} & \multirow[t]{2}{*}{ Outra } & \multicolumn{2}{|c|}{$\underline{\text { Associação }}$} & \multirow{2}{*}{$\frac{\text { Razão de Chance }}{(95 \% \text { IC) }}$} \\
\hline & & & Phi & $\mathrm{P}$ & \\
\hline Sexo & & & 0,133 & $<0,001$ & $2,51(1,57-4,02)$ \\
\hline Feminino & 56 & 344 & & & \\
\hline Masculino & 29 & 448 & & & \\
\hline Estilo de aprendizagem $(\mathrm{N}=865)$ & & & 0,108 & 0,001 & $2,19(1,34-3,58)$ \\
\hline Reflexivo & 61 & 419 & & & \\
\hline Ativo & 24 & 361 & & & \\
\hline Preferência ao ingresso $(\mathrm{N}=873)$ & & & 0,224 & $<0,001$ & $4,68(2,87-7,65)$ \\
\hline Pediatria & 32 & 90 & & & \\
\hline Outra & 53 & 698 & & & \\
\hline Preferência ao término do $1^{\circ}$ ano & & & 0,306 & $<0,001$ & - \\
\hline Primeira (Pediatria) & 33 & 63 & 0,292 & $<0,001$ & $7,30(4,40-12,12)$ \\
\hline Segunda & 13 & 94 & & & \\
\hline Terceira & 12 & 104 & & & \\
\hline Indeterminada & 27 & 527 & & & \\
\hline Nível de rendimento (Ped./Cir.) & & & 0,070 & 0,038 & $1,61(1,02-2,53)$ \\
\hline Maior & 49 & 363 & & & \\
\hline Menor & 36 & 429 & & & \\
\hline Monitoria em Pediatria & & & 0,306 & $<0,001$ & $8,03(4,81-13,40)$ \\
\hline Sim & 33 & 58 & & & \\
\hline Não & 52 & 734 & & & \\
\hline Estágio seletivo & & & 0,687 & $<0,001$ & $85,48(45,07-162,13)$ \\
\hline Pediatria & 70 & 41 & & & \\
\hline Outro & 15 & 751 & & & \\
\hline Rendimento global & & & 0,073 & 0,031 & $1,65(1,04-2,61)$ \\
\hline Maior & 52 & 387 & & & \\
\hline Menor & 33 & 405 & & & \\
\hline Época da graduação & & & $-0,129$ & $<0,001$ & $0,40(0,24-0,64)$ \\
\hline 1994-2001 & 60 & 386 & & & \\
\hline $2002-2007$ & 25 & 406 & & & \\
\hline
\end{tabular}


Tabela 2

Medidas de correlação entre índice de interesse iterativo em Pediatria e sexo, estilo de aprendizagem e escolha de residência em Pediatria

\begin{tabular}{lcccccc}
\hline $\begin{array}{l}\text { Índice de interesse em } \\
\text { Pediatria }\end{array}$ & $\begin{array}{c}\text { Sexo } \\
\text { Feminino }\end{array}$ & Masculino & Reftilo $(b)$ & \multicolumn{2}{c}{ Residência $(c)$} \\
\hline 0 & 244 & 377 & 328 & 289 & Sim & Não \\
\hline 1 & 75 & 65 & 84 & 53 & 29 & 111 \\
2 & 50 & 23 & 39 & 33 & 18 & 513 \\
3 & 18 & 5 & 19 & 4 & 17 & 6 \\
4 & 13 & 3 & 10 & 6 & 13 & 3 \\
\hline
\end{tabular}

Notas: 1 Pontuação $(1=\operatorname{sim}, 0$ = não): preferência por ocasião do ingresso + preferência após o primeiro ano + monitoria em Pediatria + estágio seletivo na área.

2 Medidas de correlação (tau-b; significância): a $(0,212 ;<0,001) ; \mathrm{b}(0,073 ; 0,024)$; c $(0,469 ;<0,001)$.

Os egressos foram também classificados em quatro grupos conforme o perfil de adesão, em termos de precocidade ou não, e confirmação ou não de preferência por Pediatria. O primeiro grupo (A) inclui os egressos que manifestaram atração inicial (preferência por ocasião do ingresso e/ou após o primeiro ano) e escolheram Pediatria na residência; o segundo grupo (B) inclui aqueles que não manifestaram atração inicial, mas escolheram a especialidade; o terceiro grupo (C) inclui os egressos que manifestaram atração inicial, mas não escolheram a especialidade; 0 quarto grupo (D) inclui os que nem manifestaram atração inicial, nem escolheram Pediatria na residência médica.

A Tabela 4 revela as diferenças na distribuição de indicadores de atributos pessoais ou relacionados ao curso entre 873 egressos distribuídos nos quatro grupos. Observou-se associação positiva entre cada indicador e a classificação no perfil de adesão, com tendência crescente na força da associação segundo a ordem temporal de ocorrência dos indicadores relacionados ao curso. A magnitude foi menor e não significante para nível de rendimento $(P h i=0,085 \mathrm{p}=0,098)$, intermediária e significante para monitoria $(P h i=0,369 p<0,001)$, maior e muito significante para estágio seletivo ( $P h i=0,687 p<0,001)$. Houve, entretanto, diferenças nas magnitudes de associação entre classificação de adesão e indicadores curriculares em dois aspectos: quanto à época de graduação (as associações foram mais fracas para os egressos de 2002 a 2007) e quanto ao sexo dos participantes (as associações foram mais fortes para os egressos do sexo feminino).
Os grupos nessa classificação de adesão diferiram significativamente também em relação às médias de três índices acadêmicos: rendimento nas disciplinas de Pediatria, razão de rendimento (Pediatria/Cirurgia) e rendimento global no curso. As médias foram superiores, nos três índices, para os egressos (grupos A + B) que escolheram Pediatria na residência médica.

Quais das características selecionadas se relacionaram com os quatro indicadores de interesse no caminho para a escolha de Pediatria? Análises sequenciais de regressão logística revelaram - fixando consecutivamente cada indicador de interesse como variável de desfecho - os seguintes respectivos antecedentes, na ordem temporal, como fatores preditivos:

1. Para preferência por ocasião do ingresso, o fator preditivo foi sexo feminino;

2. Para preferência ao término do primeiro ano, os fatores preditivos foram preferência por ocasião do ingresso e sexo feminino;

3. Para monitoria em Pediatria, os fatores preditivos foram época de graduação (1994-2001), preferência por ocasião do ingresso, sexo feminino e rendimento na disciplina;

4. Para estágio seletivo em Pediatria, os fatores preditivos foram monitoria na área, época de graduação (1994-2001), preferência ao término do primeiro ano e estilo reflexivo de aprendizagem.

Por fim, análises de regressão logística foram realizadas com inclusão dos nove indicadores constantes da Tabela 1 como va- 
Tabela 3

Medidas de associação entre indicadores pessoais e curriculares e grupos de perfil de adesão ao campo da Pediatria (atração inicial v. escolha de residência médica) entre egressos de Medicina da Universidade de Brasília $(\mathrm{N}=873)$

\begin{tabular}{|c|c|c|c|c|c|c|}
\hline \multirow{2}{*}{$\begin{array}{l}\text { Indicadores } \\
\text { Progressão temporal }\end{array}$} & \multicolumn{4}{|c|}{ Grupos $^{a}$} & \multicolumn{2}{|c|}{$\underline{\text { Associação }}$} \\
\hline & A & B & C & $\mathrm{D}$ & $\mathrm{Ph}$ & $\mathrm{P}$ \\
\hline Sexo & & & & & 0,250 & $<0,001$ \\
\hline Feminino & 30 & 26 & 77 & 267 & & \\
\hline Masculino & 8 & 21 & 34 & 410 & & \\
\hline Estilo de aprendizagem & & & & & 0,113 & 0,012 \\
\hline Reflexivo & 27 & 34 & 63 & 356 & & \\
\hline Ativo & 11 & 13 & 46 & 315 & & \\
\hline Nível de rendimento & & & & & 0,085 & 0,098 \\
\hline Maior & 25 & 24 & 52 & 308 & & \\
\hline Menor & 13 & 23 & 59 & 369 & & \\
\hline Monitoria em Pediatria & & & & & 0,369 & $<0,001$ \\
\hline Sim & 21 & 12 & 20 & 37 & & \\
\hline Não & 17 & 35 & 91 & 640 & & \\
\hline Estágio seletivo & & & & & 0,687 & $<0,001$ \\
\hline Pediatria & 32 & 38 & 5 & 36 & & \\
\hline Outra área & 6 & 9 & 106 & 641 & & \\
\hline
\end{tabular}

(a) Grupos: A (atração inicial sim, escolha sim); B (atração inicial não, escolha sim); C (atração inicial sim, escolha não); D (atração inicial não, escolha não).

riáveis independentes e a inscrição para residência médica como variável de desfecho. As variáveis foram incluídas em quatro blocos, na ordem temporal de ocorrência dos indicadores. Este modelo de análise conservou sete fatores preditivos independentes para a escolha de Pediatria: sexo, estilo de aprendizagem, ordem de preferência pela especialidade, monitoria na área, rendimento global no curso, estágio seletivo em Pediatria e biênio de graduação ( 1 = primeiro, 7 = último). Os indicadores preferência por ocasião do ingresso e nível de rendimento não emergiram como fatores preditivos no modelo de análise. Dois fatores, sexo e monitoria, não mostraram nível de significância na predição do desfecho. O modelo classificou corretamente
94,91\% da totalidade das escolhas (70,59\% das opções por Pediatria e $97,56 \%$ de quaisquer outras opções).

Os fatores preditivos significantes representam indicadores curriculares em ordem temporal (estágio seletivo em Pediatria, rendimento global no curso), indicadores pessoais (estilo de aprendizagem e ordem de preferência por Pediatria) e de conjuntura histórica (biênio da graduação). A Tabela 5 resume os dados da análise. A magnitude do efeito, calculada a partir da razão de chance (odds ratio ${ }^{33}$, é maciça para opção de estágio seletivo (eta $\left.{ }^{2}=0,628\right)$, média para rendimento global (eta $\left.{ }^{2}=0,162\right)$ e pequena para estilo de aprendizagem $\left(\right.$ eta $\left.^{2}=0,070\right)$ e ordem de preferência $\left(\right.$ eta $\left.^{2}=0,017\right)$. 
Tabela 4

Diferenças nas médias (desvios padrão) de três índices de rendimento dos grupos de perfil de adesão ao campo da Pediatria (atração inicial v. escolha de residência médica) entre egressos de Medicina da Universidade de Brasília $(\mathrm{N}=873)$

\begin{tabular}{lcccccc}
\hline Indicadores & \multicolumn{2}{c}{ Grupos $^{a}$} & \multicolumn{3}{c}{$\underline{\text { Anova }}^{\mathrm{b}}$} \\
Índices de rendimento & $\mathrm{A}$ & $\mathrm{B}$ & $\mathrm{C}$ & $\mathrm{D}$ & $\mathrm{F}$ & $\mathrm{P}$ \\
\hline Rendimento em Pediatria & $4,13(0,37)$ & $3,98(0,31)$ & $3,98(0,37)$ & $3,92(0,37)$ & 4,97 & 0,002 \\
Razão de rendimento & $1,13(0,20)$ & $1,06(0,15)$ & $1,04(0,15)$ & $1,04(0,16)$ & 3,78 & 0,010 \\
(Pediatria/Cirurgia) & & & & & & \\
Rendimento global & $4,25(0,32)$ & $4,19(0,28)$ & $4,12(0,34)$ & $4,11(0,31)$ & 3,30 & 0,020 \\
\hline
\end{tabular}

(a) Grupos: A (atração inicial sim, escolha sim); B (atração inicial não, escolha sim); C(atração inicial sim, escolha não); D (atração inicial não, escolha não).

(b) Análise de variância $(\mathrm{N}=873, \mathrm{df}=3)$.

Tabela 5

Modelo de regressão logística para fatores preditivos da escolha de residência médica em Pediatria (Ped.) por egressos de Medicina da Universidade de Brasília ( $\mathrm{N}=865)$

\begin{tabular}{|c|c|c|c|c|}
\hline $\begin{array}{l}\text { Fatores preditivos } \\
\text { (ordem temporal) }\end{array}$ & Coef. B & Erro padrão & Significância & $\begin{array}{l}\text { Razão de chance } \\
\qquad(\mathrm{IC}=95 \%)\end{array}$ \\
\hline $\operatorname{Sexo}(F / M)$ & 0,62 & 0,37 & 0,0939 & $\begin{array}{c}1,86 \\
(0,99-3,83)\end{array}$ \\
\hline Estilo de aprendizagem (R/A) & 1,01 & 0,39 & 0,0087 & $\begin{array}{c}2,75 \\
(1,29-5,87)\end{array}$ \\
\hline Preferência por Pediatria & 0,48 & 0,15 & 0,0013 & $\begin{array}{c}1,62 \\
(1,21-2,17)\end{array}$ \\
\hline Monitoria em Pediatria & 0,77 & 0,45 & 0,0861 & $\begin{array}{c}2,16 \\
(0,90-5,21)\end{array}$ \\
\hline Rendimento global & 1,59 & 0,60 & 0,0079 & $\begin{array}{c}4,92 \\
(1,52-15,94)\end{array}$ \\
\hline Estágio seletivo em Pediatria & 4,70 & 0,43 & $<0,0001$ & $\begin{array}{c}110,20 \\
(47,06-258,06)\end{array}$ \\
\hline Biênio de graduação (1 a 7) & 0,14 & 0,06 & 0,0077 & $\begin{array}{c}1,15 \\
(1,03-1,29)\end{array}$ \\
\hline (Constante) & $-13,44$ & 2,79 & $<0,0001$ & - \\
\hline
\end{tabular}

Notas: 1 Variável de desfecho: escolha ou não de Pediatria na inscrição para programa de residência médica (R1).

2 Fator excluído: razão de rendimento em Pediatria. 


\section{DISCUSSÃO}

A procura de residência médica constitui atualmente um passo crítico que visa não somente à especialização de competências, mas à própria adequação às condições do trabalho profissional. A escolha da área conclui, muitas vezes, um longo processo decisório que envolve alternativas de confirmação, eliminação ou inclusão de uma especialidade de preferência. O processo incorpora uma busca de compatibilidade pessoal em face dos domínios e desafios de diferentes opções de carreira, e dos atributos e atividades dos respectivos profissionais ${ }^{11,17}$. Essa concepção sugere que a conscientização precoce de preferências serviria ao processo preliminar de circunscrição ou projeção de opções de carreira, que envolve percepções reais, ainda que imprecisas, dos atributos e demandas em jogo e sua similaridade com fatores de personalidade, interesses e habilidades do estudante ${ }^{5}$.

Os dados do estudo mostram que houve confirmação da atração inicial (por ocasião do ingresso e/ou ao término do primeiro ano) para 44,7\% dos egressos que escolheram Pediatria na residência, um achado congruente com indicações da literatura sobre o impacto dessa predisposição. Interesses específicos, sexo e traços peculiares de personalidade têm sido associados à preferência por Pediatria em trabalhos internacionais. No grupo de atração inicial ficou evidente a relação entre persistência da preferência por Pediatria e os motivos subjacentes de gosto e disposição para lidar com crianças. A relação estreita e significante entre sexo feminino e preferência inicial presumivelmente foi incorporada em indicadores subsequentes de interesse em Pediatria. Estudo recente demonstra que a importância atribuída ao atendimento integral do paciente medeia o efeito de sexo na intenção de seguir uma área generalista na profissão médica ${ }^{34}$.

O estilo de aprendizagem, na forma caracterizada no estudo, provavelmente é um componente do perfil psicológico de personalidade ${ }^{35}$. Assim, a associação entre o estilo reflexivo e a escolha da especialidade ganha relevância; denota, possivelmente, um enfoque de interação mais centrado na pessoa do que em procedimentos nesse grupo de participantes. Cabe ressaltar, aliás, o nítido contraste na valência desse indicador como fator preditivo independente na escolha da residência: enfoque reflexivo (estilo divergente, especialmente) no caso da Pediatria e enfoque ativo (estilo convergente, especialmente) na opção por Cirurgia $^{36}$.

O montante de $18 \%$ dos egressos mudou de preferência no tocante à Pediatria do início ao término do curso, com 12,6\% eliminando essa opção para a qual houvera atração inicial, e outros 5,4\% incluindo Pediatria em substituição a uma atração inicial diversa. A eliminação e a inclusão de preferências foram proces- sos gradativos ao longo da graduação e quase consolidados quando da opção por estágio seletivo no internato, segundo os dados da Tabela 3. Fatores históricos (desistência proporcionalmente maior nos biênios recentes), pessoais (maior desistência entre mulheres e maior inclusão entre aqueles de estilo reflexivo de aprendizagem) e curriculares (declínio no interesse, já expresso no menor rendimento) contribuíram para o abandono da atração inicial. Registre-se que, entre os egressos que desistiram de Pediatria, a maioria (59\%) manteve preferência por área clínica e fez inscrição para residência em Clínica Médica (44,5\%) ou Ginecologia e Obstetrícia (14,5\%).

Vivências curriculares e do ambiente de treinamento em serviço no rodízio de internato, além de influenciarem a confirmação ou eliminação da preferência inicial, possivelmente encorajaram a inclusão de Pediatria como escolha final para residência médica numa parcela substancial dos egressos. São bem sugestivas dessa vertente de influências vivenciais as relações da escolha da especialidade com o rendimento relativo nas disciplinas, o exercício de monitoria e o estágio seletivo na área.

O exercício da monitoria vale como marcador do processo de decisão do participante, na medida em que reflete seu interesse pela disciplina e sua expectativa de interagir com preceptores que possam servir de exemplo profissional e orientá-lo. No caso, a experiência da monitoria na área foi significantemente associada ao sexo feminino, à atração inicial por Pediatria e ao rendimento absoluto e relativo na disciplina, apesar da limitação da oferta e da restrição do tempo disponível para os estudantes no quinto e sexto anos do curso. Embora não sendo fator preditivo independente da escolha da residência, o exercício da monitoria foi preditivo da escolha de estágio seletivo em Pediatria.

Como seria de esperar por causa da posição temporal na trajetória da graduação, o estágio seletivo no terceiro semestre do internato foi o fator preditivo com maior magnitude de efeito na escolha. Essa fase do treinamento em serviço constitui uma ocasião decisiva para o formando verificar sua compatibilidade pessoal com a especialidade em termos de conteúdo, contexto de trabalho, tipos de problemas clínicos confrontados e relação interpessoal com clientes e profissionais da área. Ajudaria, assim, na confirmação ou invalidação das expectativas projetadas por interesses, estudos e vivências anteriores (inclusive no próprio internato), conforme indicado pela relação da opção de estágio seletivo com experiência de monitoria na disciplina, preferência inicial pela especialidade e estilo de aprendizagem reflexivo.

Além das trajetórias de predisposição e de influência vivencial, a identificação do rendimento global como fator preditivo 
significante sugere uma terceira vertente, de caráter estratégico, na escolha da residência em Pediatria. O prospecto de classificação para vagas na residência seria maior, em tese, para candidatos com perfil acadêmico superior e compatibilidade pessoal com a área, mormente para graduandos do sexo feminino.

Observou-se associação negativa entre recentidade da graduação e escolha da especialidade (Tabela 1). A elevação da frequência de desistências, sem compensação de novas adesões nos três últimos biênios, relacionou-se possivelmente a duas dimensões de influência: aspectos relacionados ao curso (incluindo circunstâncias institucionais desfavoráveis) e aspectos prospectivos, provenientes da antevisão das perspectivas de inserção profissional. Assim, um aspecto intrigante nos achados da análise de regressão logística foi a identificação da época de graduação recente como fator preditivo positivo da escolha. $\mathrm{Na}$ análise sequencial, houve inversão do sentido de associação da variável biênio de graduação após inclusão de eventos curriculares no modelo de regressão logística. A inversão talvez represente fatores ocultos, não expressos em indicadores curriculares, em conexão com expectativas idiossincráticas dos graduandos recentes.

A constatação da queda na adesão à Pediatria no período 2002-2007, junto com a evidência mais recente de declínio na própria atração inicial pela área, põe em foco a questão da formação do pediatra e o que fazer no aconselhamento dos estudantes quanto à opção da carreira profissional. Vários fatores de impacto potencial no recrutamento têm sido apontados na literatura, envolvendo questões tanto da ambiência de educação e treinamento quanto de oportunidades e recompensas no trabalho médico ${ }^{29}$. A Sociedade Brasileira de Pediatria tem realçado a questão da valorização profissional e sua conexão com a melhoria da qualidade do atendimento integral da criança e do adolescente ${ }^{37}$.

Em suma, as limitações deste estudo incluem a singularidade da escola médica e a restrição na amostra de influências potenciais, quanto aos condicionamentos do estudante e suas razões, bem como quanto aos fatores de atração ou repulsão na realidade das condições da prática profissional ${ }^{38}$. Fatores ocultos poderiam explicar falsos negativos e positivos no modelo de predição da escolha. No aspecto metodológico, valem também as precauções quanto à fidedignidade de inferências causais no uso de regressão logística.

Os pontos fortes do estudo incluem a perspectiva longitudinal dos dados, do ingresso do estudante à conclusão do curso, o tamanho significativo da população-alvo ensejado pelo amplo número de coortes consecutivas e a reduzida perda (menos de $2 \%$ ) de casos ou dados. Os resultados ilustram diferentes confi- gurações de interesse e adesão, sugestivas de variação individual na trajetória da escolha da carreira. Realçam também a importância das oportunidades educacionais para orientar o estudante e implicam a força oculta da conjuntura institucional e social na influenciação sobre decisões de carreira. $\mathrm{O}$ uso da regressão logística, a par da economia na identificação de fatores significantes na predição da escolha, demonstrou razoável sensibilidade e boa especificidade nos índices de acerto.

Em conclusão, o estudo revelou ao menos oito fatores de influência na escolha de residência médica em Pediatria por egressos do curso de Medicina da UnB durante um período de 14 anos. Os achados sugerem três diferentes vertentes de interessee influência no processo de escolha. Um modelo de regressão logística identificou cinco fatores preditivos significantes, incluindo atributos pessoais (estilo de aprendizagem e ordem de preferência) e eventos curriculares (estágio seletivo na área profissional e rendimento acadêmico no curso), bem como a fase da conjuntura histórica (época de graduação) na série de coortes consecutivas.

\section{AGRADECIMENTOS}

Os autores agradecem a todos os egressos do curso de Medicina, reconhecendo a cooperação e a generosidade da participação no levantamento de expectativas.

\section{REFERÊNCIAS}

1. Bland CJ, Meurer LN, Maldonado G. Determinants of primary care career choice: a non-statistical meta-analysis of the literature. Acad Med. 1995;70:620-641.

2. Dohn H. Choices of career in medicine: some theoretical and methodological issues. Med Educ. 1996; 30:157-160.

3. Kassebaum D, Szenas PL, Schuchert MA. Determinants of the generalist career intentions of 1995 graduating medical students. Acad Med. 1996;71:197-209.

4. Sobral DT. Escolha de carreira em medicina por graduandos da Universidade de Brasília. Brasília Médica. 2000; 37:8-13.

5. Petrides KV; McManus IC. Mapping medical careers: questionnaire assessment of career preferences in medical school applicants and final-year students. BMC Med Educ. 2004;4:18

6. Meurer LN. Influence of medical school curriculum on primary care specialty choice: analysis and synthesis of the literature. Acad Med. 1995; 70:388-397.

7. Senf JH, Campos-Outcalt D, Watkins AJ, Bastacky S, Killian C. A systematic analysis of how medical school charac- 
teristics relate to graduates' choices of primary care specialties. Acad Med. 1997;72:524-533.

8. Xu G, Veloski J, Barzansky B, Hojat M, Diamond J, Silenzio VMB. Comparisons among three types of generalist physicians: personal characteristics, medical school experiences, financial aid, and other factors influencing career choice. Adv Health Sci Educ. 1997;1(3):197-207.

9. Ellsbury KE, Carline JD, Irby DM, Stritter FT. Influence of third-year clerkships on medical student specialty preferences. Adv Health Sci Educ. 1998;3:177-186.

10. Zeldow PB, Preston RC, Daugherty SR. The decision to enter a medical specialty: timing and stability. Med Educ. 1992;26:327-332.

11. Burack JH, Irby DM, Carline JD, Ambrozy DM, Ellsbury KE, Stritter FT. A study of medical students' specialty choice pathways: trying on possible selves. Acad Med. 1997;72:534-541.

12. Maiorova T, Stevens F, Scherpbier A, Zee J. The impact of clerkships on students` specialty preferences: what do undergraduates learn for their profession? Med Educ. 2008;42:554-562.

13. Figueiredo JFC, Rodrigues MLV, Troncon LEA, Cianflone ARL. Influence of gender on specialty choices in a Brazilian medical school. Acad Med. 1997;72:68-70.

14. Coffin SE, Babbott D. Early and final preferences for pediatrics as a specialty: a study of U.S. medical school graduates in 1983. Acad Med. 1989;64:600-605.

15. Carraccio CL, Englander R, Baffa JM, Berman MA. Choosing pediatrics as a career: influences and trends. Clin Pediatr. 1999;38:179-181.

16. Doucet H, Shah MK, Cummings TL, Kahn MJ. Comparison of internal medicine, pediatric, and medicine/pediatrics applicants and factors influencing career choices. South Med J. 1999; 92:296-299.

17. Senf JH, Kutob R, Campos-Outcalt D. Which primary care specialty? Factors that relate to a choice of family medicine, internal medicine, combined internal medicine-pediatrics, or pediatrics. Fam Med. 2004; 36: 123-130.

18. Turner G, Lambert TW, Goldacre MJ, Turner S. Career choices for paediatrics: national surveys of graduate of 1974-2002 from UK medical schools. Child Care Health Dev. 2007; 33: 340-346.

19. Miller AJ. Pediatrics. In: Freeman B The Ultimate Guide to Choosing a Specialty. ${ }^{\text {th }}$ ed. New Delhi: McGraw-Hill; 2007. p. 359-375.
20. Duke University School of Medicine. Pathway evaluation program for medical professionals. [acesso em 10/9/2008]. Disponível em: www.smbs.buffalo.edu/resident/CareerCounseling/.

21. Borges NJ, Savickas ML. Personality and medical specialty choice: a literature review and integration. Journal Career Assessment. 2002;10: 362-380.

22. Vaidya NA, Sierles FS, Raida MD, Fakhoury FJ, Przybeck TR, Cloninger CR. Relationship between specialty choice and medical student temperament and character assessed with Cloninger inventory. Teach Learn Med. 2004;16:150-156.

23. Markert RJ, Rodenhauser P, El-Baghdadi MM, Juskaite K, Hillel A, Maron BA. Personality as a prognostic factor for specialty choice: a prospective study of 4 medical school classes. Medscape J Med. 2008; 10(2):49.

24. Bitran M, Zuniga D, Lafuente M, Viviani P, Mena B. Influencia de la personalidad y el estilo de aprendizaje en la elección de especialidad médica. Rev Med Chil. 2005;133:1191-1199.

25. Sobral DT. Estilos de aprendizagem de estudantes de medicina e suas implicações. Rev Bras Educ Méd. 2005;29:5-12.

26. Kaplowitz PB, Boyle R, Russell MA, Jiandong L. The effect of pediatric clerkship on medical students`attitudes towards pediatrics at eleven medical schools. Acad Med. 1996;150:435-439.

27. Al-Asnag MA, Jan MMS. Influence of clinical rotation on intern attitudes toward pediatrics. Clin Pediatr 2002; 41: 509-514.

28. Antommaria AHM, Firth SD, Maloney CG. Evaluation of an innovative pediatric clerkship structure using multiple outcome variables including career choice. J Hosp Med. 2007;2:401-408.

29. Kanto WP. Influencing career decisions in pediatrics. J Pediatr. 2004; 144: 693-694.

30. Sobral DT. Escolha de carreira em medicina e internato eletivo: um estudo de coortes. Rev Hosp Clin Fac Med São Paulo. 1998;53:325-329.

31. Kolb DA. Learning style inventory technical manual. Boston: McBer; 1976.

32. Howell DC. Statistical Methods for Psychology. $5^{\text {th }}$ ed. Pacific Grove (CA): Duxbury; 2002.

33. Tabachnick BG, Fidell LS. Using Multivariate Statistics. $5^{\text {th }}$ ed. Boston: Pearson; 2007. p. 437-505.

34. Behrend TS, Meade AW, Newton DA, Grayson MS. Measuring invariance in careers research. Using IRT to study 
gender differences in medical students`specialization decisions. Journal of Career Development. 2008;35:60-83.

35. Furnham A. Personality and learning style - A study of 3 instruments. Pers Indiv Differ 1992; 13: 429-438.

36. Sobral DT. Influences on choice of surgery as a career: a study of consecutive cohorts in a medical school. Med Educ. 2006;40:522-529.

37. Sociedade Brasileira de Pediatria. SPB propõe mudanças na residência em pediatria. (Acesso em 17/10/2008). Disponível em: www. sbp.com.br.

38. Conselho Federal de Medicina. O Médico e o seu Trabalho. Brasília: CFM; 2004.

\section{CONTRIBUIÇÃO DOS AUTORES}

Os autores assumem e responsabilidade pública pelo conteúdo do artigo, tendo participado de forma suficiente na análise e inter- pretação dos dados assim como na redação deste texto. Fizeram a revisão da versão final deste artigo e o aprovaram antes do encaminhamento para a publicação. O autor Dejano T. Sobral foi responsável pela concepção e desenho do estudo, iniciadoem 1988.

\section{CONFLITO DE INTERESSES}

Declarou não haver

\section{ENDEREÇO PARA CORRESPONDÊNCIA}

Dejano T. Sobral

Universidade de Brasília - Faculdade de Medicina

Brasília

CEP. 70919-970 - Cx.P 04569 DF

E-mail: dtsobral@gmail.com 